\title{
Destructive aortic valve endocarditis from Brucella abortus: survival with emergency aortic valve replacement
}

\author{
JOSEPH C CLEVELAND, RAYMOND J SUCHOR, AND JOHN DAGUE \\ From the Departments of Cardiovascular and Thoracic Surgery and Cardiology, Carle Clinic, \\ 602 West University, Urbana, Illinois 61801, USA
}

Cleveland, J C, Suchor, R J, and Dague, J (1978). Thorax, 33, 616-618. Destructive aortic valve endocarditis from Brucella abortus: survival with emergency aortic valve replacement. Brucella abortus infection of the aortic valve caused acute aortic regurgitation leading to severe left ventricular failure in a 62-year-old man. He made an excellent recovery after emergency aortic valve replacement. This is the third reported case of successful heart valve replacement for Brucella endocarditis and the second such case involving the aortic valve.

Brucellosis is an acute or subacute infectious disease that lasts for weeks or months. Its manifestations are fever, chills, sweating, weakness, and muscle and joint discomfort. Death is rare, but infective endocarditis is the most common cause. Calcific aortic valvular disease is usually present, but the infection may occur on a normal valve. Surgical treatment for destruction of a heart valve by endocarditis is a well-established procedure (Stason et al, 1968; Stinson et al, 1976). Nevertheless, because of the rarity of Brucella endocarditis only two cases of surgical treatment of the condition could be found after a thorough search (Wolfe et al, 1973; O’Meara et al, 1974). We report the third case.

\section{Case report}

The patient, an American civil engineer and restaurant owner who had lived in Equador since 1945, presented to the Carle Clinic in May 1977 complaining of fatigue. He was found to have hacmaturia, but radiological examination of the urinary tract showed no cause. Anaemia was also no:ed $(\mathrm{Hb} 10 \cdot 1 \mathrm{~g} / \mathrm{dl})$. The patient was seen again on 26 June, when he returned feeling quite ill. An interim history of fever and joint swelling was obtained. He was admitted to hospital, where he was found to be dyspnoeic and orthopnoeic. Soft systolic and diastolic aortic murmurs were heard. The sedimentation rate was 62 , the blood urea 39 $\mathrm{mg} / 100 \mathrm{ml}$, and serum creatinine $2 \cdot 3 \mathrm{mg} / 100 \mathrm{ml}$.
Haematuria persisted. Because bacterial endocarditis was suspected, multiple blood cultures were obtained. Digoxin and frusemide were administered to control the heart failure, and a course of ampicillin and gentomycin was started.

The patient rapidly developed severe left ventricular failure from aortic regurgitation. A cardiac surgical consultation was obtained on the fourth day. Emergency aortic valve replacement was recommended.

On 1 July 1977, emergency aortic valve replacement was performed. Before the operation, oxacillin, cephalothin, and streptomycin were given. Inspection of the aortic valve at aortotomy showed multiple vegetations with a perforation of the noncoronary cusp and destruction of the left and right coronary cusps over about $40 \%$ of their surface area. Cultures of the vegetative material were obtained. The valve was totally excised and replaced by a $23 \mathrm{~mm}$ glutaraldehyde-preserved porcine aortic stent-mounted heterograft.*

Recovery from the operation was slow but uneventful, and the patient was discharged after 18 days. He has continued to make satisfactory progress. Cultures of the vegetations showed a slow growing, pleomorphic, Gram-negative organism, which was quite sensitive to all antibiotics. After the operation the patient was treated with oxacillin and streptomycin for five days, and then penicillin for four weeks. When the identity of the organism (Brucella abortus) was confirmed by the bacteri*Edwards Laboratories, Santa Ana, California, USA. 
ology section of the State of Illinois Department of Health, tetracycline was given for two weeks. Antibiotics were then discontinued. The striking clinical improvement is illustrated by the immediate preoperative chest radiograph (fig 1) and the radiograph six weeks later (fig 2 ).

\section{Discussion}

Brucellosis in humans is rare in the USA, although it remains a considerable problem in animals. In 1973 the lowest number of cases in the US was reported (175 cases). In 1976 there were 268 cases (JAMA 1977). The mortality rate is less than $1 \%$, but endocarditis is usually the cause of death.

Most cases of primary endocarditis are caused by the Streptococcus or the Staphylococcus. Even prosthetic valve endocarditis with its more unusual organisms is rarely due to $B$ abortus (Stinson et al, 1976). We were able to find only two other cases in which the successful treatment of $B$ endocarditis necessitated heart valve replacement (Wolf et al, 1973; O'Meara et al, 1974).

In the case report of O'Meara et al (1974), the
Brucella infection affected a prosthetic StarrEdwards mitral valve, and this was replaced.

The case of Wolf et al (1973) most closely resembles our case. A 44-year-old man was operated on under similar conditions to our patient. Aortic valve replacement with a Starr-Edwards prosthesis led to a remarkable recovery. It should be noted that the bacteriological diagnosis in this case was based on the serum agglutinin titre and Gram stain. The organism was never cultured from the patient's blood or aortic valve. The first surgeon to report valve replacement for $B$ endocarditis was Ehrenhaft, but the prosthetic valve also became infected, leading to the patient's death after 38 days (Ehrenhaft, 1967).

The inclusion of streptomycin in the operative antibiotic regimen was probably a factor in preventing reinfection, since streptomycin and tetracycline are the antibiotics most effective against Brucella species. Use of a porcine glutaraldehydepreserved valve may also help, for residual glutaraldehyde in small concentration remains for a time in the sewing ring of the prosthetic valve and may inhibit initial bacterial growth while neoendothelialisation occurs.

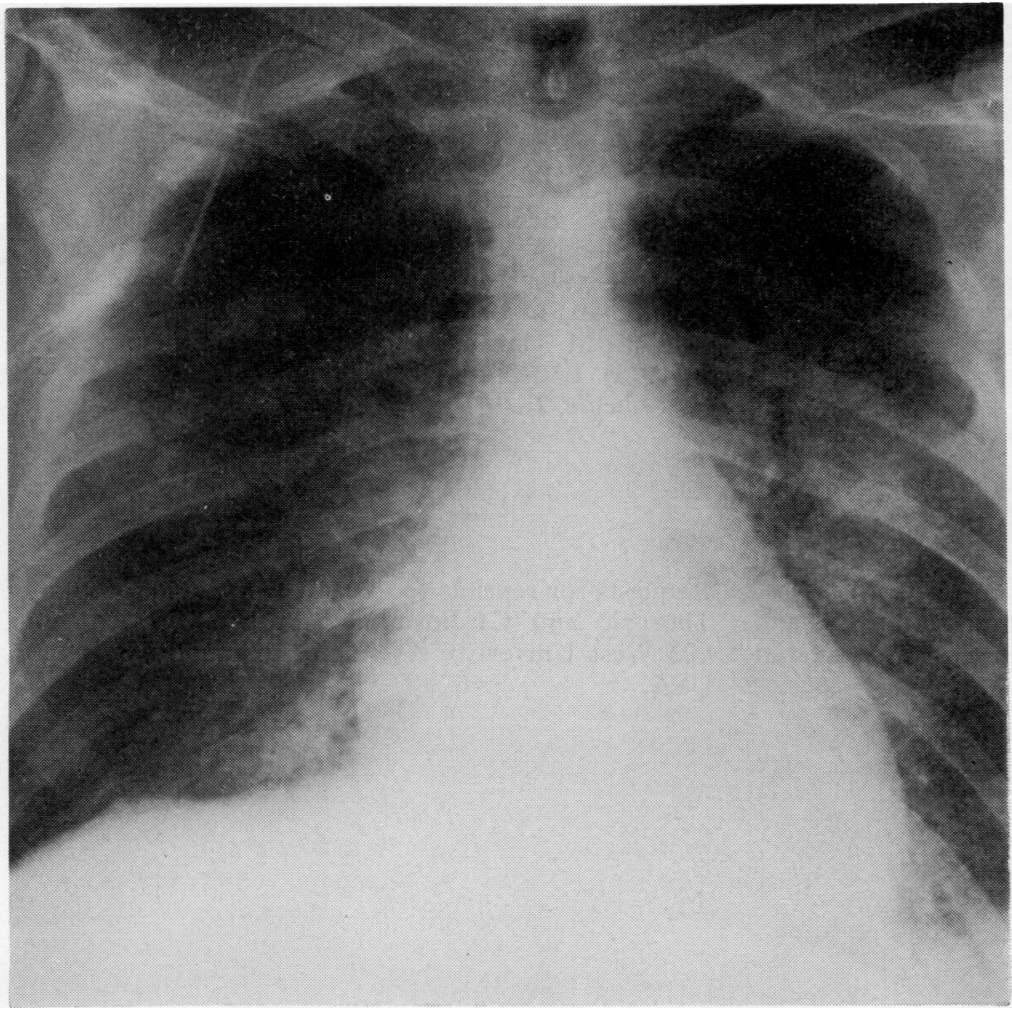

Fig 1 Chest radiograph immediately before operation showing severe pulmonary vascular congestion and oedema. 


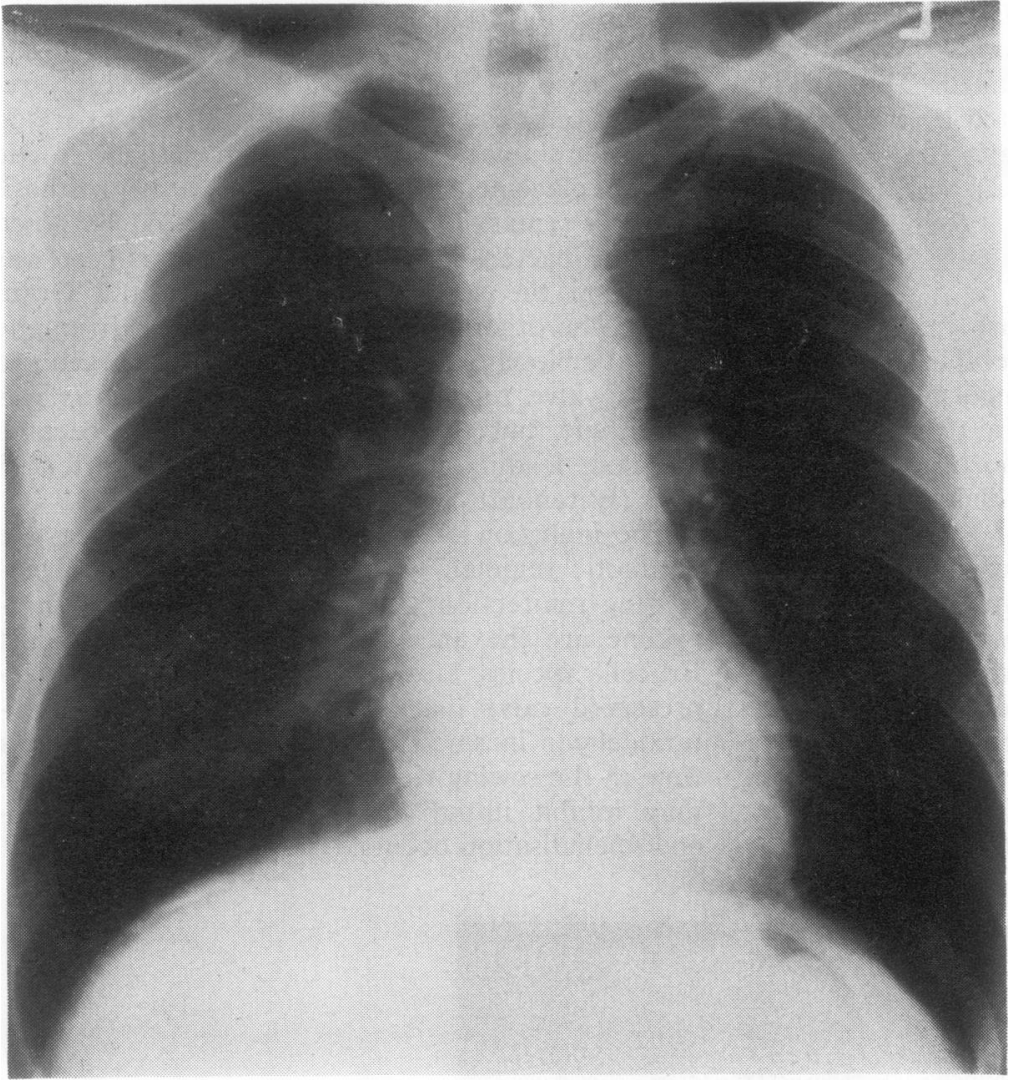

Fig 2 Chest radiograph six

\section{References}

Ehrenhaft, J L (1967). In discussion of a paper by Kaiser, G C, et al. Valve replacements in cases of aortic insufficiency due to active endocarditis. Journal of Thoracic and Cardiovascular Surgery, 54, 491.

Medical News Section (1977). Journal of the American Medical Association, 238, 302.

O'Meara, J B, Eykyn, S, Jenkins, B S, Braimbridge, M V, and Phillips, I (1974). Brucella melitensis endocarditis: successful treatment of an infected prosthetic mitral valve. Thorax, 29, 377-381.

Stason, W B, De Sanctis, R W, Weinberg, A N, and Austen, W G (1968). Cardiac surgery in bacterial endocarditis. Circulation, 38, 514-523.
Stinson, E B, Griepp, R B, Vosti, K, Copeland, J G, and Shumway, N E (1976). Operative treatment of active endocarditis. Journal of Thoracic and Cardiovascular Surgery, 71, 659-665.

Wolf, L M, Hazan, E, Courtois, H, and Schrub, C L, (1973). Endocardite brucellienne. La Nouvelle Presse Medicale, 2, 493-495.

Requests for reprints to: Dr J C Cleveland, Section of Thoracic and Cardiovascular Surgery, Carle Clinic, $\mathcal{N}$ 602 West University Avenue, Urbana, Illinois 61801, 6 USA. 